\title{
SPIN REORIENTATION AND EXCHANGE COUPLING IN THE $\mathrm{Dy}_{1-x} \mathrm{Er}_{x} \mathrm{Fe}_{10} \mathrm{Si}_{2}$ COMPOUNDS*
}

\author{
P. Stefański, A. Kowalczyk, D. Skoryna, A. Szlaferek \\ AND A. WRZECIONO
}

\author{
Institute of Molecular Physics, Polish Academy of Sciences \\ Smoluchowskiego 17/19, 60-179 Poznań, Poland
}

\begin{abstract}
The structural and magnetic properties of $\mathrm{Dy}_{1-x} \mathrm{Er}_{x} \mathrm{Fe}_{10} \mathrm{Si}_{2}$ are investigated. X-ray analysis reveals that these compounds are of the tetragonal ThMn12 structure. In this structure the rare earth atoms occupy one crystallographic position 2(a). The unit cell contains 26 atoms. The spin reorientation temperature, $T_{\mathrm{SR}}$, was measured from the temperature dependence of the initial susceptibility using an ac bridge of mutual inductance of the Harsthorn type. $\mathrm{Dy}^{+3}$ and $\mathrm{Er}^{+3}$ have opposite contributions to the entire magnetic anisotropy. The spin reorientation temperature is found to be about $271 \mathrm{~K}$ in $\mathrm{DyFe}_{10} \mathrm{Si}_{2}$ and $48 \mathrm{~K}$ in $\mathrm{ErFe}_{10} \mathrm{Si}_{2}$. The values are discussed applying the crystal field model. The value of the rare earth-transition metal exchange coupling constant $J_{\mathrm{RFe}} / k_{\mathrm{B}}$ derived from the mean-field model analysis of the Curie temperature is about $-13 \mathrm{~K}$. The $\mathrm{Fe}-\mathrm{Fe}$ exchange integral is much higher and is equal to about $75 \mathrm{~K}$.
\end{abstract}

PACS numbers: $75.30 . \mathrm{Gw}, 75.50 . \mathrm{Gg}$

\section{Introduction}

Recently, many groups of researchers have investigated the iron-rich ternary compounds of the type $R(F e, T)_{12}$ in which $R$ is a rare earth element and $T$ is a stabilizing element such as titanium, vanadium, chromium, silicon, etc. All these compounds crystallize in the $\mathrm{ThMn}_{12}$ type of structure [1]. In this structure the $\mathrm{R}$ atom occupies one kind of crystallographic site, ie. 2(a), the other atoms occupy $8(\mathrm{i}), 8(\mathrm{j})$ and $8(\mathrm{f})$ positions.

In the rare earth-transition metal compounds $(\mathrm{R}-\mathrm{T})$, the contribution to the net anisotropy from the $R$ sublattice generally dominates at lower temperatures, whereas the T-sublattice anisotropy dominates at high temperatures. Also, the R-sublattice anisotropy decreases much faster with increasing temperature than the T-sublattice anisotropy. In the $\mathrm{RFe}_{10} \mathrm{Si}_{2}$ compounds, the $\mathrm{Fe}$ anisotropy favourizes the $c$-axis in the whole range of temperature [2]. The easy magnetization

*This work was supported by the grant no. 202039101 of the Committee for Scientific Research. 
direction (EMD) of the $R$ sublattice depends on the crystal electric field (CEF) interaction and the exchange field experienced by the $R$ ion.

In this paper the competition between the opposite contributions of different rare earth sublattices to the entire anisotropy is investigated. For this purpose we used two ions with different signs of second-order Stevens factors: $\operatorname{Dy}\left(\alpha_{J}<0\right)$ and $\operatorname{Er}\left(\alpha_{J}>0\right)$.

\section{Experimental}

The samples were prepared by induction melting of stoichiometric amounts of the constituents in a water-cooled boat under atmosphere of argon. The ingots were inverted several times to insure homogeneity. Then the samples were wrapped in a Ta foil, sealed in a quartz tube filled with argon and annealed for two weeks at $900^{\circ} \mathrm{C}$ and then rapidly cooled to room temperature. X-ray diffraction was performed on powdered samples using $\mathrm{Cu} K_{\alpha}$ radiation. Diffraction patterns showed only characteristic lines of the $\mathrm{ThMn}_{12}$ structure.

The spin reorientation (SR) temperature, $T_{\mathrm{SR}}$, was determined from measurements of the temperature dependence of the initial susceptibility using an ac bridge of mutual inductance of the Harsthorn type. The intensity of alternating field used was $20 \mathrm{~A} / \mathrm{m}$ and frequency $-15 \mathrm{~Hz}$.

The Curie temperature was determined by measuring the temperature dependence of magnetization $(M)$ under a weak external field. The value of $T_{\mathrm{C}}$ was obtained by plotting $M^{2}$ versus $T$ and extrapolating to $M^{2}=0$.

\section{Results and discussion}

X-ray diffraction patterns revealed that the compounds crystallize in the tetragonal $\mathrm{ThMn}_{12}$-type structure.

The $T_{\mathrm{C}}$ values decrease when Dy atoms are replaced by Er ones (Table). This decrease in $T_{\mathrm{C}}$ may be related to the reduction of the lattice parameters with the increasing $\mathrm{Er}$ content. Because most of the $\mathrm{Fe}-\mathrm{Fe}$ distances in $\mathrm{RFe}_{10} \mathrm{Si}_{2}$ compounds are less than $2.5 \AA$, a decrease in lattice parameters would mean a decrease in $\mathrm{Fe}-\mathrm{Fe}$ distance and consequently, results in reduction of the exchange interactions and a decrease in $T_{\mathrm{C}}$.

In order to derive information regarding the magnetic coupling constants $J_{\mathrm{FeFe}}$ and $J_{\mathrm{RFe}}$ between the rare earth (R) and Fe moments in the $\mathrm{RFe}_{10} \mathrm{Si}_{2}$ compounds, we have performed a standard mean-field analysis of the observed Curie temperatures. In this method $T_{\mathrm{C}}$ can be expressed as

$$
3 k_{\mathrm{B}} T_{\mathrm{C}}=a_{\mathrm{FeFe}}^{2}+\left(a_{\mathrm{FeFe}}^{2}+4 a_{\mathrm{RFe}} a_{\mathrm{FeR}}\right)^{1 / 2},
$$

where

$$
\begin{aligned}
& a_{\mathrm{FeFe}}=Z_{\mathrm{FeFe}} J_{\mathrm{FeFe}} S_{\mathrm{Fe}}\left(S_{\mathrm{Fe}}+1\right), \\
& a_{\mathrm{RFe}} a_{\mathrm{FeR}}=Z_{\mathrm{RFe}} Z_{\mathrm{FeR}} S_{\mathrm{Fe}}\left(S_{\mathrm{Fe}}+1\right)\left(g_{J}-1\right)^{2} J(J+1) J_{\mathrm{RFe}}^{2} .
\end{aligned}
$$

Equation (1) is obtained after neglecting weak R-R interactions. From the crystal structure of $\mathrm{ThMn}_{12}$, it follows that each $\mathrm{R}$ atom has an average number 


\section{TABLE}

Curie temperatures and spin-reorientation temperatures of the $\mathrm{Dy}_{1-x} \mathrm{Er}_{x} \mathrm{Fe}_{10} \mathrm{Si}_{2}$ compounds.

\begin{tabular}{l|c|c}
\hline \hline$x$ & $T_{\mathrm{C}}[\mathrm{K}]$ & $T_{\mathrm{SR}}[\mathrm{K}]$ \\
\hline 0.0 & 568 & 271 \\
0.25 & 562 & 218,134 \\
0.50 & 557 & 207 \\
1.0 & 549 & 48
\end{tabular}

$Z_{\mathrm{RFe}}=17 \mathrm{Fe}$ nearest neighbours while each $\mathrm{Fe}$ atom has an average number of $Z_{\mathrm{FeR}}=2 \mathrm{R}$ nearest neighbours and an average number of $Z_{\mathrm{FeFe}}=8 \mathrm{Fe}$ neighbours. $S_{\mathrm{Fe}}=0.65$ is the quasi-spin of the Fe ions. Using the experimental data of $T_{\mathrm{C}}\left(535 \mathrm{~K}\right.$ for $\mathrm{YFe}_{10} \mathrm{Si}_{2}$ [2]) one finds $J_{\mathrm{FeFe}} / k_{\mathrm{B}}=75 \mathrm{~K}$. This value of the coupling constant was subsequently used in Eq. (1) when applied to $\mathrm{DyFe}_{10} \mathrm{Si}_{2}\left(T_{\mathrm{C}}=568 \mathrm{~K}\right)$ and $\mathrm{ErFe}_{10} \mathrm{Si}_{2}\left(T_{\mathrm{C}}=549 \mathrm{~K}\right)$. This leads to the values of $J_{\mathrm{RFe}} / k_{\mathrm{B}}$ equal to $13 \mathrm{~K}$ and $12 \mathrm{~K}$ for $\mathrm{DyFe}_{10} \mathrm{Si}_{2}$ and $\mathrm{ErFe}_{10} \mathrm{Si}_{2}$, respectively.

The value of the uniaxial anisotropy constant $K_{1}$ due to the rare earth sublattice is given by the equation [3]

$$
K_{1}=(-3 / 2) \alpha_{J}\left\langle r^{2}\right\rangle A_{2}^{0}\left\langle O_{2}^{0}\right\rangle+5 \beta_{J}\left\langle r^{4}\right\rangle A_{4}^{0}\left\langle O_{4}^{0}\right\rangle+(-21 / 2) \gamma_{J}\left\langle r^{2}\right\rangle A_{6}^{0}\left\langle O_{6}^{0}\right\rangle .
$$

It is valid in this class of compounds in the large exchange limit [4]. $\alpha_{J}, \beta_{J}, \gamma_{J}$ are the Stevens factors [5], $\left\langle r^{n}\right\rangle$ are Hartree-Fock radial integrals [6], $A_{n}^{m}$ are crystal electric field potentials. $O_{n}^{m}$ are Stevens equivalent operators [4].

The magnitude and sign of $K_{1}$ is mainly determined by the first term in Eq. (4). Knowing that $A_{2}^{0}<0$ [7] in this class of compounds for the rare earth with $\alpha_{J}<0(\mathrm{Nd}, \mathrm{Tb}, \mathrm{Dy}, \mathrm{Ho})$, the rare earth anisotropy constant $K_{1}$ has a negative sign (when neglecting higher order terms in Eq. (4)) and for these ions SR transition should appear. For $\mathrm{DyFe}_{10} \mathrm{Si}_{2} T_{\mathrm{SR}}=271 \mathrm{~K}$ (see Table). For $\mathrm{Er}^{+3}$ ion $\left(\alpha_{J}>0\right)$ SR transition appears as a result of domination of the second and third term in Eq. (4). In this class of compounds for $\mathrm{Er}^{+3}$ ion the higher order crystal field terms has a remarkable influence on the anisotropy [8].

In $\mathrm{Dy}_{1-x} \mathrm{Tm}_{x} \mathrm{Fe}_{10} \mathrm{Si}_{2}$ samples there exist two rare earth sublattices each of them manifesting SR transition. A "mixing" of SR takes place. It appears that for $x=0.25$ the SR at $T_{\mathrm{SR}}=134 \mathrm{~K}$ comes from Er-sublattice. The $\mathrm{Er}$ anisotropy is enhanced by the Dy planar anisotropy. SR at $218 \mathrm{~K}$ comes from Dy-sublattice. While lowering Dy content $T_{\mathrm{SR}}$ coming from Dy-sublattice shifts to lower temperatures and as a result of competition of Dy- and Er-sublattice anisotropies with axial Fe-sublattice anisotropy SR transition is produced at $207 \mathrm{~K}$.

\section{References}

[1] D.B. de Mooij, K.H.J. Buschow, Philips J. Res. 42, 246 (1987).

[2] P. Stefański, A. Wrzeciono, J. Magn. Magn. Mater. 82, 125 (1989). 
262 P. Stefański, A. Kowalczyk, D. Skoryna, A. Szlaferek, A. Wrzeciono

[3] P.-A. Lindgård, O. Danielsen, Phys. Rev. B 11, 351 (1975).

[4] P. Stefański, A. Wrzeciono, Phys. Status Solidi B 151, K195 (1989).

[5] M.T. Huthings, Solid State Phys. 16, 277 (1964).

[6] A.J. Freeman, R.E. Watson, Phys. Rev. 127, 2058 (1962).

[7] K.H.J. Buschow, D.B.de Mooij, M. Brouha, H.H.A. Smit, R.C. Thiel, IEEE Trans. Magn. MAG-24, 227 (1964).

[8] P. Stefański, A. Kowalczyk, Solid State Commun. 77, 397 (1991). 